\title{
Autonomous driving manoeuvres in urban road traffic environment: a study on roundabouts
}

\author{
Pérez Joshué*, Milanés Vicente*, de Pedro Teresa* and Vlacic Ljubo** \\ * AUTOPIA program, Centre for Automation and Robotics (CAR), University Polytechnic of Madrid (UPM) \\ - Spanish National Research Council (CSIC), Madrid, Spain
}

(e-mail: \{joshue.perez, vicente.milanes,teresa.pedro\}@car.upm-csic.es)

**Intelligent Control Systems Laboratory, Griffith University, Brisbane, Australia (e-mail: 1.vlacic@griffith.edu.au).

\begin{abstract}
The highway and urban traffic safety has significantly been improved thanks to advanced development of Intelligent Transportation Systems technologies. However, some driving aspects have remained outside of this evolution or they are still in the development stage. Autonomous vehicle performance on roundabouts is one of these. In this paper, a simple and practical approach for the driverless control on roundabouts is described. Firstly, a description of a path planning is explained. Finally, a new concept for lateral control on roundabouts is introduced, taking into account entrances, exits and lane changes inside the roundabouts. The experiments have been tested in a $3 \mathrm{D}$ simulator that emulates the behaviour of driverless vehicle from the real world, - Cybercars. The results are promising, and leave the door open for future implementation in real vehicles.
\end{abstract}

Keywords: Lane keeping, autonomous vehicles, roundabouts, digital map, Intelligent Transportation Systems (ITS).

\section{INTRODUCTION}

In the last two decades, the roundabouts have progressively become a more popular alternative to tackling intersection problems. However, at the same time they have become a point of conflict for many drivers (Abaza and Hussein, 2009). Studies (in the U.S and China) have shown that drivers that don't have experience using roundabouts cause traffic jams in the vicinity (Xiaoguang et al, 2004; Abaza and Hussein, 2009; Yu et al, 2009).

Roundabouts with and without traffic-signal controls have been intensely studied and widely used (Xiaoguang et al, 2004). Nevertheless, so far, roundabout applications only consider the management problem, speed control, geometrical characteristics and even flow problems ( $\mathrm{Yu}$ et al, 2009; Molinete et al, 2009; Hong-bo et al, 2008; Linjun and Chao, 2009). However, completely autonomous vehicle control has still not been considered in roundabouts. With the geometrical conditions of each roundabout (center position in 2 dimensional Cartesian coordinate system- and radius) we can construct a map using the parametric equations. The generated map is used as reference for a cascade control scheme (proposed in Fig. 1) for the lateral control on an autonomous vehicle.

A roundabout can be considered as a composition of several intersections (Molinete et al, 2009). Hence, the control over a roundabout could bring near to small segments in a circle. Some solutions just try to read a map from a previous loaded file and then try to control the vehicle. Since each roundabout has a particular radius, entrance road and exits, in this work we present a solution for the trajectory dynamic generation in a roundabout. Moreover, a control scheme that permits to follow the generated trajectory, using a driverless vehicle steering wheel on the entrance, during and exiting of a roundabout, is proposed. Finally, the control in a single and two circulatory lanes roundabout is considered.

In this work, a new approach for lateral control in a roundabout, considering a circle parametric equation to determinate the path to be followed for the vehicle, has been developed. The entrance, exits, path planning and even lane change inside the roundabout have been considered. A double gain controller is proposed to solve the lane keeping problem in a roundabout. The proposed algorithm has been tested in a 3D simulator, which provides real time data (GPS and lidar information) of the vehicle.

The rest of the paper is organized as follows. A description of the control system, as well as the world requirements used to generate the trajectory, and the simulator is explained in section 2. Section 3 explains the experiments carried out in simulation, considering the subroutines used for controlling before, during and after the roundabout. A discussion section is presented in section 4. And finally the conclusions and future works are explained in section 5 .

\section{CONDITIONS AND CONTROL IN ROUNDABOUTS.}

There are many types of roundabouts. For instance: highway junctions usually have large roundabouts, from two to six lanes around the centre; urban intersections sometimes have a radius of less than two meters and some may have traffic lights regulating the flow. Therefore, it is important to narrow 
the range of actions of the controller in the roundabouts proposed in this work. The requirements are:

- The smallest radius in the roundabout is conditioned for a turning radius of the vehicle and the lane width. A Cybercar has 2.5 meters of turning radius, and the lane width is established at 3 meters. Consequently, the minimum radius is 4 meters, considering that the vehicle would go over the circulatory lane. However, for applications with real vehicles (larger than a Cybercar) 6 meters would be the minimum radius.

- Only two-lane roundabouts are used in the experiments. Although, one and two entrances and exit lanes are considered.

- The number of entrances and exits are limited to four. Perpendicular roads to the tangent line in the roundabout are usually used. But, also different degrees of inclination at entrances and exits are considered.

- One vehicle is used. Cooperation with other vehicles will be considered in future works.

- The speed is set. Roundabout signal will send the reference speed to the vehicle, which can change inside the roundabout. The position of the signal is known. The control proposed in this paper is over the steering wheel.

- Communication with a central station or a beacon at the roundabout has been considered for receiving world information. Only the radius and the roundabout centre are needed for our control algorithm.

Fig. 1 shows the control scheme proposed in this work. It considers sensor information, location and dimension of the roundabout. It also allows a change of the exit (previously defined) once the vehicle is inside the roundabout. There are two important points in the autonomous steering control in roundabouts: the route to follow and the controller to carry out. In this section, detailed descriptions of the route (trajectory generator), the control system (double gain controller) and the test environment are explained.

\subsection{Perception stage}

Driving into a roundabout is preceded and followed as per straight road driving. When the vehicle detects a roundabout ahead (i.e.: reading a traffic signal) the trajectory generator is activated. It is in charge of collecting data from the environment, signalling the next reference point to the control stage. Fig. 1 illustrates the trajectory generator blocks, from the inputs (Sensors, world information and HMI blocks) to the dynamic map generator. Below, a description of the conditional inputs is given.

- Sensor information: it provides the information obtained through on-board sensors: GPS and lidar sensors.

It is compatible with UTM coordinates used in real vehicle implementations with DGPS. The lidar information is used to stop the vehicle when there is an obstacle in front of the vehicle. It will also be used in future works for the purpose of driving in roundabouts in cooperation with other vehicles. Other sensors can also be added.

- Features of the roundabout: only the radius and the coordinates are needed to generate the map. Information about routes (before and after), traffic signal, and other possible exits in the roundabout can be read using a XML file as is proposed in (Furda and Vlacic, 2009).

- Human Machine Interface (HMI): the driverless control is programmed to follow a predefined route. However, a HMI was developed to test the control proposed in this paper. The user can change the lane in the roundabout, as well as select another exit once inside the roundabout.

The input information gives the route and the global position of the vehicle to the perception stage of the roundabout control algorithm (Fig. 1). Below are the three main blocks:

- Route definer: when the vehicle is in straight segments, the route planner reads the predefined point on the map from a XML file. The route to cover by the CyberCar is loaded at the beginning of the manoeuvre among the different map files. The route definer sends the current reference position to the definer of the coordinate system. The centre of a roundabout is considered as an intersection point with a radius. Later the dynamic map generator is in charge to build the roundabout points. Finally, this block sets the target speed for the vehicle, but if a speed traffic signal is detected, the speed is changed.

- Definer of coordinate system: the information from the sensors comes in abscissa and ordinate axes, according to the simulator, or in the UTM coordinates from a real DGPS. Nevertheless, to define variables with respect to the actual street, a new reference system is needed. This considers the initial and final point of a straight segment as its ends, and the first point is the new origin in our Euclidean space. This new coordinate system draws a line between these points, which are later used to calculate the variables of our control system. These variables are recalculated in real time. When a new point is read (or generated) from the map, a new reference segment is loaded.

- Dynamic map generator: is active when the vehicle detects a roundabout. First, the equation 1, an estimation of the first angle $\alpha$ is done (Fig. 2). This is the reference (start) angle on the roundabout. In turning roundabout experiments (section 4 ) this angle is defined between $-\pi$ and $\pi$. When we increase this angle (or decrease for hand left drive countries), an estimation of the next map point is done, considering the parametric equations of the circle (eq. 1 and eq. 2 ).

$$
\begin{array}{ll}
X=\text { Radius }^{*} \cos (\alpha)+\text { Centre_abscissa } & \text { eq. } 1 \\
Y=\text { Radius }{ }^{*} \operatorname{sen}(\alpha)+\text { Centre_ordinate } & \text { eq. } 2
\end{array}
$$

Fig. 2 shows all the variables, points and roads used in the dynamic map generator in roundabouts. The tangent to the circle is just an approach to calculate the variables of the control system. A projection of the line allows us to measure the variables with less error, because if the points are close the measurement error of variables is big.

\subsection{Control stage}

The control stage is in charge of generating the variables that the feedback system has produced. Afterwards, it gives the reference to the steering wheel of the vehicle. Each block of this phase is implemented as follows: 


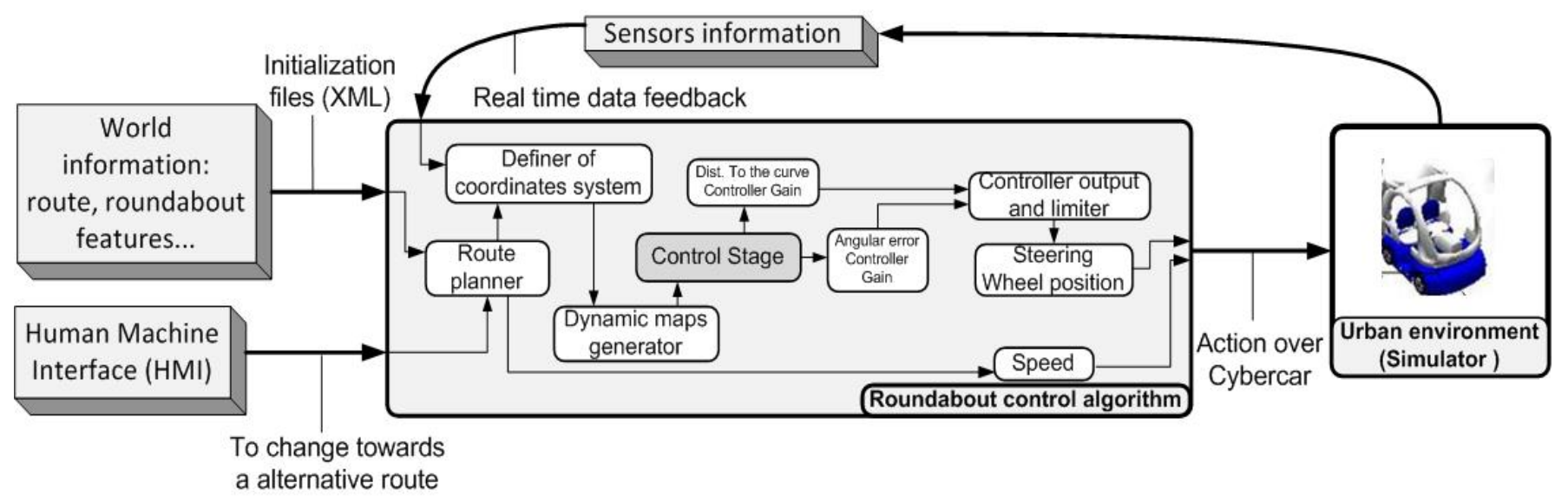

Fig. 1. Control system for autonomous vehicles in roundabouts.

- Control stage block: this block receives the information according to the line reference system (the street in straight segment, and the line tangent on the roundabout). There are two variables that feed two independent controllers: the distance to the curve and the angular error. Fig. 3 shows the variables regarding a curve line in the $3 \mathrm{D}$ simulator environment, as well the requirements to calculate these (previous and next point of the map, and the tangent line). Later, the output of each controller is averaged and limited in the controller output and limiter block (Fig. 1). Each controller works as follows:

o Distance to the curve gain controller: the distance to the curve is measured using the position of the vehicle and its point over the curve (circle). It is negative when the vehicle is on the left side of the reference and vice versa (the origin of the Cartesian system is at the beginning of the segment -Definer of coordinate system). A gain controller was added to the lane keeping. However, the lateral kinematic of the vehicle means that the higher the gain the bigger the oscillation, and the lower the gain, the slower the lateral displacement. For this reason, another controller was added to compensate to this effect.

- Angular error gain controller: this controller reduces the oscillation, and allows a faster and softer output. The control variable is the angular error. It is positive when the nose of the vehicle is to the left side of the reference line, and vice versa. The angular error is calculated according to the tangent line of the curve.

- Controller output and limiter: Both variables, Distance to the curve and Angular error, have been used by several authors in previous works [Naranjo et al, 2005; Pérez et al, 2011] for lane keeping control, in curves and straight segments using fuzzy logic controllers. However, in this paper a double gain controller is proposed to attack the automatic steering wheel control in roundabouts. For this aim, the distance to the curve and the angular error are equivalent to a Proportional and Integral part of a double PI, since the oscillations of the CyberCar are comparable with the first variable, and the steady-stage error with the second one. Before the generation of the output, both gain controllers are added and weighted according to the limits of the range of action of the steering wheel [-0.5 0.5] (Boisse et al, 2007).

Different gains have been used for straight segment and in the roundabout. These controllers can be extended to other autonomous vehicles, just by adjusting the controller gains and the output limiters.

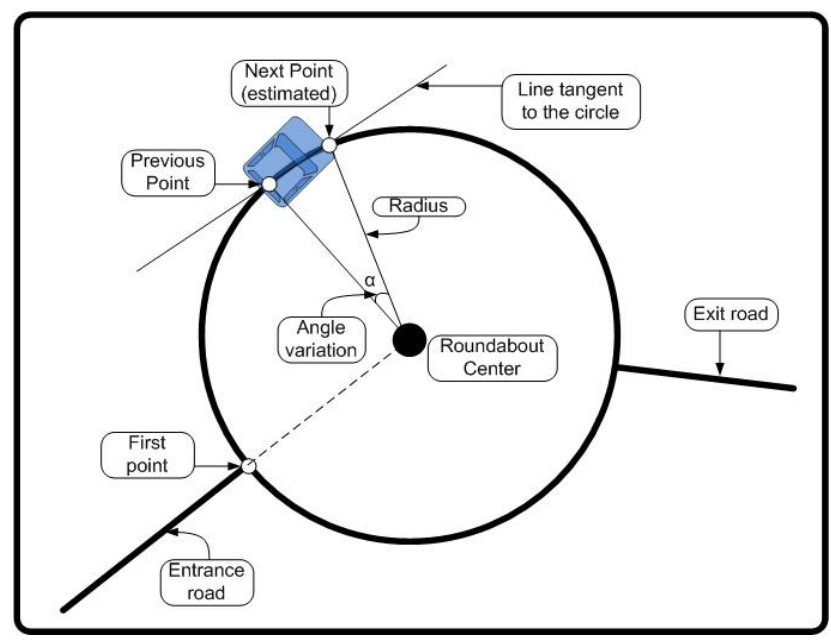

Fig. 2. Point, roads and variables used in the dynamic calculation on the roundabout.

\subsection{Action stage}

The designed control system has been tested in a dynamic virtual 3D environment. The main purpose of this simulation is to test functionalities of autonomous driving algorithms in city-like road traffic situations (Boisse et al, 2007). Fig. 3 shows the 3D simulator based on the Nathan Campus (Griffth University). A realistic performance of the vehicle, which includes: physical structure, shape, collision model and mass distribution of the vehicle, as well as the engine and four independent wheels (strength, damping suspension and friction parameters), have been considered in this simulator (Boisse et al, 2007).

The driverless vehicle receives the target for the steering wheel and the speed actuator. The whole virtual environment 
considers vehicles, world model (road, building, roundabout) and moving obstacles (others vehicles, pedestrian and motorbikes) that would be used for future cooperative manoeuvres in roundabouts. Each block of the proposed algorithm is modular and interchangeable.

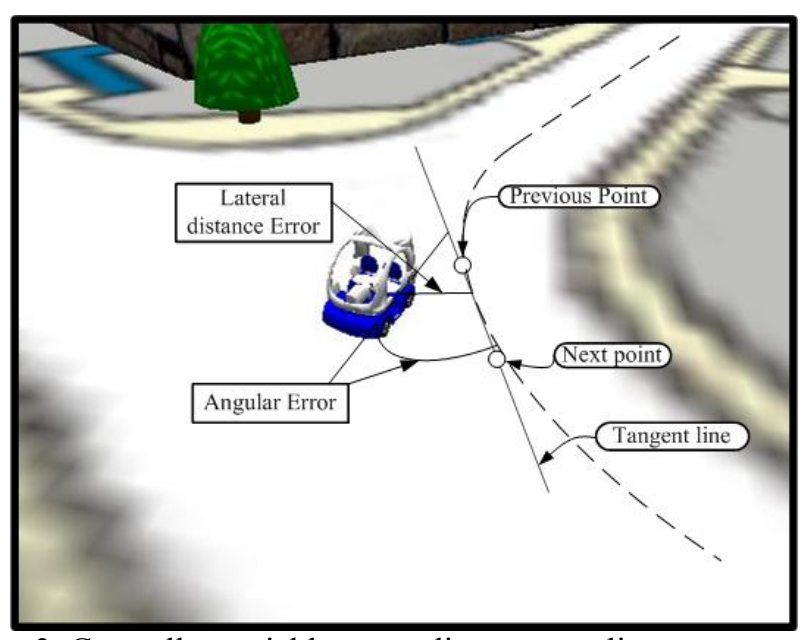

Fig. 3. Controller variables regarding a curve line

\section{EXPERIMENTS AND SIMULATIONS.}

Different experiments have been done regarding control of autonomous vehicles in roundabouts. First, lane keeping and lane change manoeuvres using several paths have been tested (before entering in the roundabout). Then, continuous turning in the roundabout, lane changes inside the roundabout (using different radii) and several dynamic map generation have been successfully made. Finally, some trials with all elements integrated into the system, entering and exiting of the roundabout, and a turning back manoeuvre within the roundabout were carried out.

\subsection{Lane keeping and lane change manoeuvres.}

Fig. 4 shows the behaviour of the vehicle when a user (from the HMI) is changing the reference lane, performing a lane change manoeuvre. This figure shows a straight segment with the new coordinate system which is using the first point of the line as the origin of the system. We can see how the system has a soft response without overshoot.

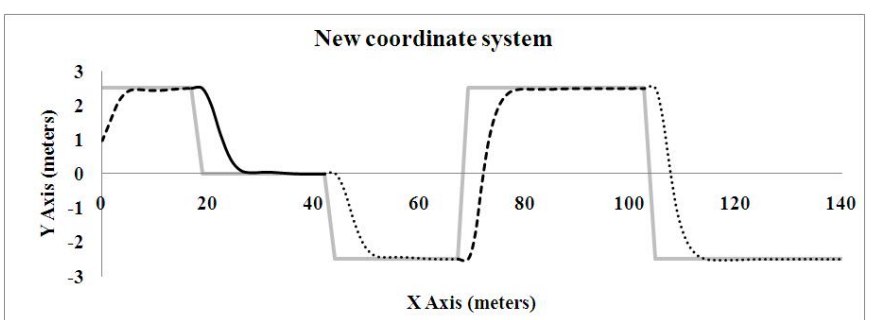

Fig. 4. Lane change.

\subsection{Turning in roundabouts .}

A dynamic generation of the roundabout is shown in Fig. 5. Three different radii have been considered: 4.5, 6 and 7.5 meters. The selection of the radii allows the verification of the design conditions. The square points are generated by our algorithm, and the straight line shows the entrance road at the roundabout. The sample generation is created every 0.2 radians.

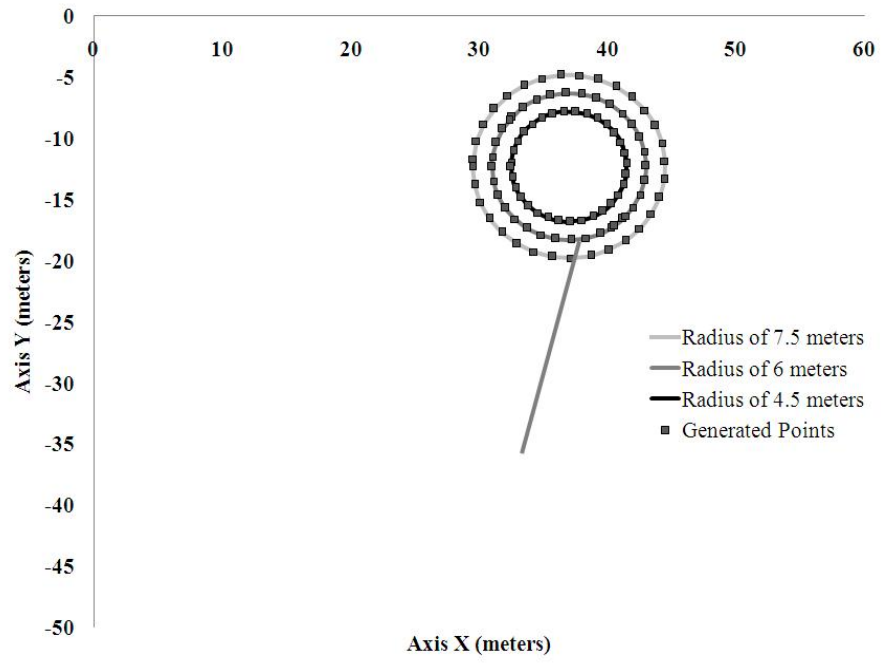

Fig. 5. Generated trajectory points on a roundabout

Fig. 6 shows the control carried out on the driverless vehicle in a roundabout. This figure shows the lane change manoeuvre implemented inside the roundabout. Some problems are found in the transient stage (passing from the roundabout of radius 6 to the other one of radius 7.5). These will be addressed in the discussion section.

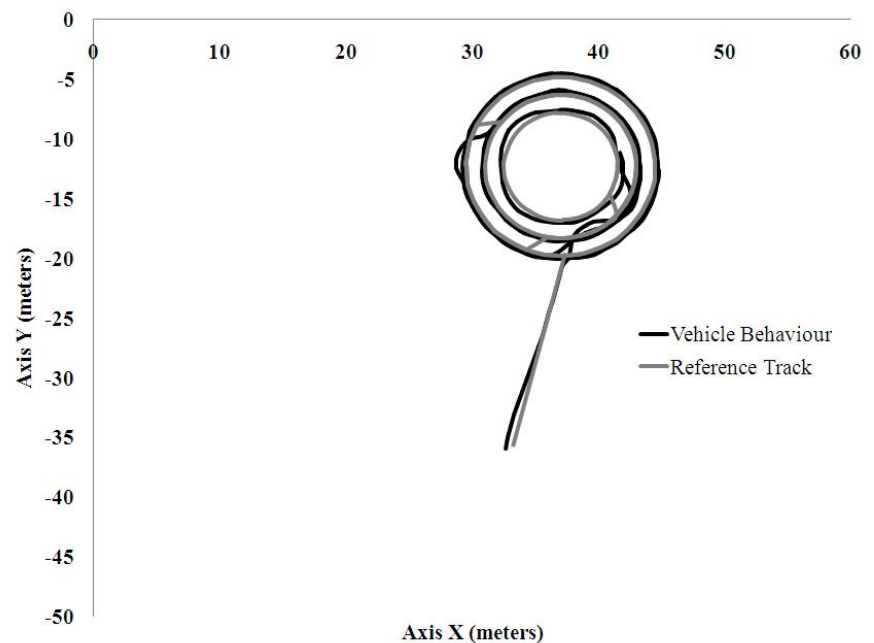

Fig. 6. Lane change in Roundabout (Radius: 4.5, 6 and 7.5 meters)

\subsection{Final test}

The last experiments carried out in this work involve the straight line control (subsection 4.1), the roundabout control (subsection 4.2) and the entrance and exit in a roundabout. The radius is set at 11 meters. Fig. 7 shows the entrance, one completed turn and the exiting of the vehicle from the roundabout. The grey points are the reference track and the black one is the vehicles behaviour. 


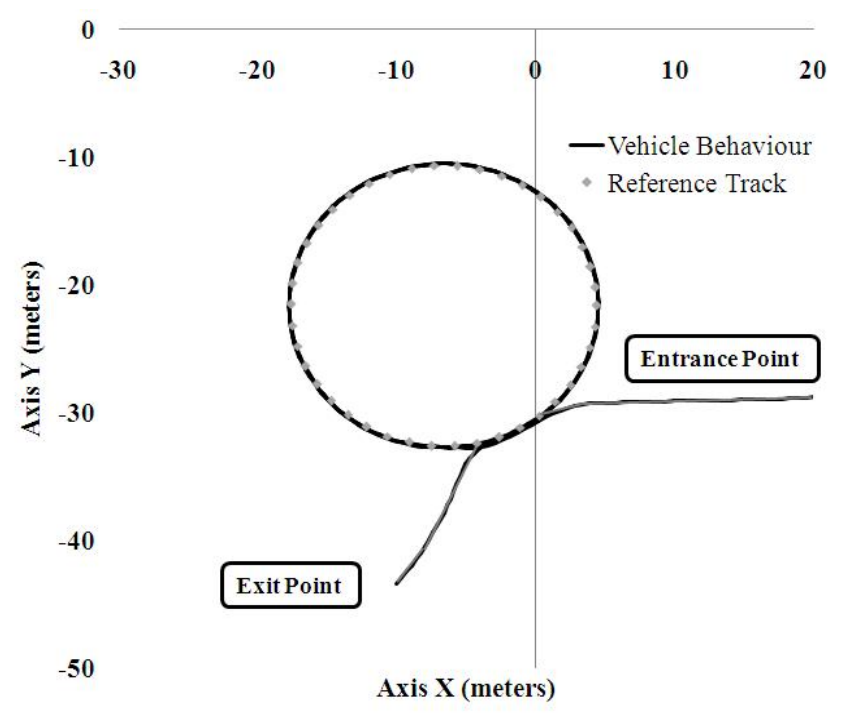

Fig. 7. Entrance and exit in a roundabout

\section{DISCUSSION}

The experiments described in this work explain the behaviour of a driverless CyberCar in a roundabout. Other authors have worked on this topic before (Xiaoguang et al, 2004; Molinete et al, 2009; Hong-bo et al, 2008), but never from the point of view of total autonomous vehicle control. In this connection, this paper presents a new control strategy in roundabouts.

First experiments (Fig. 4) show good behaviour of the vehicles in straight segments with a minimum overshoot in the transient stage (curves) when the vehicle is in the right lane, and turning right (the same with the left side). These problems are related to the bend radius at the beginning of the manoeuvre, as occurs when a human driver makes a turn. Errors are reduced when the vehicle advances on the road.

Roundabout experiments are shown in Fig. 5 and Fig. 6. The first one shows the automatic map generation using circle parametric equations. The second one shows the behaviour of the vehicle when a lane change in the roundabout is carried out. The trajectories tracking on each different radius are fairly good. The vehicle (black line) is always over the reference circle (grey line) in a steady stage. However, the error is higher when the vehicle is turning on the smallest radii ( 4.5 meter), because the steering wheel is close to its maximum capacity. Moreover, some overshoot happens when the vehicle turns right over an external radius circle. This overshoot is higher when the vehicle turns from an external circle. The continuous grey line between circles indicates when the reference (radius) changes. More attention must be paid in future works to improve the behaviour of the vehicle in these situations, though this kind of manoeuvre is unusual.

Final tests (Fig. 7) show a good performance of the CyberCar entering and exiting the roundabout. The completed turn on the roundabout in Fig. 7 shows the good control of the vehicle during the experiment. There is minimum overshoot when the vehicle is entering in the right lane. It had been solved by modifying the entering angle on the road.

\section{CONCLUSION AND FUTURE WORKS.}

The lateral control for autonomous vehicles in urban situations described in this work has shown promising results. Only considering the radius, the central coordinates and the parametric equation of a circle, an automatic map generation has been achieved. Moreover, a simple approach using two separate controllers allows driving into roundabouts without a driver. So far in literature, the lateral control for autonomous vehicles in roundabouts has not been treated. The control scheme (Fig. 1) to solve this problem is the main contribution of this work.

The simulations have permitted us to develop, as a first step, new control strategies for driving in roundabouts. This system is totally modular, permitting to exchange the different block proposed in the control scheme. In this lane, any control techniques can be easily tested.

Next step in our research will focus on the translation of the presented system to a real vehicle in a real environment. To this end, CAR' facilities that counts with a roundabout will be used to check the presented system.

Different control techniques as fuzzy or neuro-fuzzy logic controllers will be considered to improve the control proposed in this work. Moreover, the longitudinal control and cooperative manoeuvres in a roundabout will be studied. In this connection, this work constitutes a starting point to address these topics.

\section{ACKNOWLEDGMENT}

This work was carried out with the support of the Intelligent Control Systems Laboratory (ICSL), Griffith University and the Spanish projects TRÁNSITO TRA2008-06602-C03-01 Coordinación local entre vehículos e infraestructuras y GUIADE P9/08, Guiado Automático de Vehículos de Transporte Público mediante Percepción Multimodal.

\section{REFERENCES}

Abaza, O.A., Hussein, Z.S. (2009). Comparative Analysis of Multilane Roundabout Capacity "Case Study". Vehicular Technology Conference Fall IEEE 70th, pp.1-5, 20-23.

Boisse, S., Benenson, R., Bouraoui, L., Parent, M. and Vlacic, L. (2007). Cybernetic transportation systems design and development: Simulation software. IEEE ICRA'2007. Roma, Italy.

Furda, A., Vlacic, L. (2009). Towards increased road safety: Real-time decision making for driverless city vehicles. Systems, Man and Cybernetics, IEEE International Conference on , pp.2421-2426.

Hong-bo Q., Ke-ping L., Jian S. (2008). The Development and Enlightenment of Signalized Roundabout. Intelligent Computation Technology and Automation (ICICTA), International Conference on, vol.2, pp.538-542.

Linjun Y., Chao Q. (2009). Real time signal control method for multi-approach roundabouts. Management and Service Science, International Conference on, pp.1-4.

Molinete, B., Bouraui, L., Naranjo, J, Kostense, H., Hendriks, J., Alonso, J., Lobrino, R., Isasi, L.. CyberCars-2: Close Communications for Cooperation between CyberCars. Technical Report Project No IST-2004-0228062, 2009. 
Naranjo, J. E. and Gonzalez, C. and Garcia, R. and de Pedro, T. and Haber, R. E. (2005), Power-steering control architecture for automatic driving, IEEE Transactions on Intelligent Transportation Systems, vol. 6 pp 405-416.

Pérez, J., Milanés, V. and Onieva, E. (2011). Cascade Architecture for Lateral Control in Autonomous Vehicle. Intelligent Transportation Systems, IEEE Transactions on, vol. 12, no 1, pp. 73-82.

Xiaoguang Y. Xiugang L., Kun X. (2004). A new trafficsignal control for modern roundabouts: method and application. Intelligent Transportation Systems, IEEE Transactions on, vol.5, no.4, pp. 282- 287.

Yu B., Kun X., Xiaoguang Y. (2009) "Block Mechanism of Left-turned Flow at Signal-Controlled Roundabout," Intelligent Systems, WRI Global Congress on, vol.3, pp.443-449. 